\title{
A novel rate-dependent cohesive-zone model combining damage and visco-elasticity
}

\author{
Marco Musto*, Giulio Alfano \\ School of Engineering and Design, Brunel University, Kingston Lane, UB8 3PH, Uxbridge, UK
}

\begin{abstract}
This paper presents a novel rate-dependent cohesive-zone model combining damage and visco-elasticity and based on two fundamental assumptions. Firstly we postulate the existence of an intrinsic (i.e. rate-independent) fracture energy. Secondly, within a thermodynamically consistent damage-mechanics framework we assume that the evolution of the damage variable is related to the current free energy and to the intrinsic fracture energy. The underlying idea is that the energy of the bonds at the micro-level is rateindependent and that the rate-dependence of the overall dissipated energy during crack propagation is a natural by-product of the visco-elastic dissipation lumped on the zerothickness interface. Quite good agreement within an expected range of loading rates was obtained between numerical and experimental results for a DCB specimen with steel arms bonded through a rubber interface. This is despite the fact that for this application the model has been kept as simple as possible using a quadratic elastic energy and linear visco-elasticity with one relaxation time only. Therefore, the presented results support the fundamental principles behind the proposed approach and indicate that the model has the potential to be refined into a highly accurate tool of analysis based on sound physical arguments.
\end{abstract}

Keywords: interface elements, viscoelasticity, thermodynamics, damage-mechanics, crack growth in rubber, rate-dependent crack.

\footnotetext{
${ }^{*}$ Corresponding author

Email addresses: marco.musto@brunel .ac.uk (Marco Musto), giulio.alfano@brunel.ac.uk (Giulio Alfano)
} 


\section{INTRODUCTION}

Cohesive zone models stem from the seminal work by Barenblatt [1] and have often being coupled with interface elements for the purpose of modelling crack propagation along interfaces. The first application in the context of finite-element methods is due to Hilleborg [2]. The key idea of cohesive-zone modelling is to introduce a finite length process zone at the crack tip, opposed to the classical Griffith's approach where all the dissipation occurs at a singularity at the crack tip. A cohesive-zone model is characterized by a "traction-discontinuity jump" relationship, playing a role analogue to a material consitutive law for a continuum material. This relationship also provides, in the case of infinitesimal elastic deformations, the link to Linear Elastic Fracture Mechanics as its integral equals the critical energy release rate. To allow the presence on the interface of a discontinuity jump, in the sequel denoted as $\delta$, the kinematics of the problem has to be enriched relaxing the regularity requirements on the displacement field $\mathbf{u}(\mathbf{x})$, not requiring that $\mathbf{u}(\mathbf{x}) \in \mathbf{C}^{\mathbf{0}}$. It is easy to see, at least heuristically, that the cohesive-zone approach converges to Griffith's crack model as the process zone length tends to zero (a claim that has been rigorously proved by Giacomini [3]).

The rate dependence of the mechanical response leading to crack initiation and/or growth can not be neglected for a wide class of engineering applications. The complexity of the problem and the presence of numerous competing factors is evident from the fact the fracture thoughness may not show a monotonic trend with respect to crack speed, even when the latter is small enough not to consider intertial effects. Furthermore, even when such trend is monotonic, fracture toughness can increase with crack speed for some materials and decrease for others $[4,5,6]$. The importance of the problem has justified numerous experimental investigations. For example, results for a DCB test on a rubber modified epoxy show a decrease in fracture thoughness with increasing rate of applied displacement [7], whilst for a DCB made of Al 6061-T6 adherends and polyethylene as the adhesive the opposite trend has been reported [8].

Ancillary issues such as the possible sudden transition in some cases from stable to unstable propagation, also known as "stick - slip" behaviour, have been suggested to depend 
on the rate dependence of the fracture mechanism in the process zone $[9,10,11]$.

In general, the overall rate dependence can arise as a consequence of the rate dependence of the bulk material's behaviour, of the interface response itself, or of both. A number of cohesive zone models and modelling strategies have been presented covering these different assumptions.

Among the first group we will refer to Geers et al. [12], who analyzed peel testing of PET. They were able to capture the rate sensitivity of the test results by only modelling the rate dependence of the bulk material. A similar approach is followed in a study by Nguyen and Govindjee [13], who studied the propagation of a crack in an infinitely long strip of visco-elastic material. To demonstrate how the total fracture energy dissipated during crack propagation increases with the crack speed they use a cohesive-zone model with an intrinsic fracture energy so that the rate-dependence originates from the bulk material only. As examples of the second approach a reference is made to $\mathrm{Xu}$ et al. [8, 14] who similarly constructed a rate-dependent model by adding the contributions from a rate-independent and a rate-dependent element, the latter given by a Maxwell element. This quite satisfactorily replicates a class of experimental results but suffers from some inconsistencies in the formulation such as the fact that the traction discontinuosly goes to zero after reaching a threshold displacement given by a critical separation. Corigliano et al. [15] also focus on the rate-dependence of the interface to model a DCB carbon fibre-Poly-Ether-Imide (PEI) specimen. The interface deterioration is reproduced using two alternative phenomenological approaches, one of them based on softening plasticity and a second one in which a rate-dependent damage evolution law is adopted. Another example of this type of approach is the model proposed by Allen and Searcy [16], in which the interface element is conceived through an homogenization procedure conducted at the micromechanical scale.

Finally, Liechti et al. [17] introduced rate-dependent behaviour in both the bulk material and the interface, motivating their decision by observing significant differences in crack surface depending on test speed, which convinced them of the necessity of modelling the viscous losses at the interface. They also assumed the interface strength could 
be assimilated to a non-linear elastic response summed to a viscous contribution, given by a non-newtonian dashpot. Damage evolution is implicit in their formulation as the elastic response is given by a bilinear elastic traction-separation law. Furthermore, the authors suggest that the use of a non-newtonian dashpot could possibly reproduce the rate-dependent nature of void formation. The model proposed by Hutchinson, Pardoen and Landis [18] follows the same approach of introducing the rate-dependence in both the bulk and the interface. They are able to investigate the competing effects and present an explanation for the possible non-monotonic relationship between crack speed and toughness. The rate dependence of the cohesive zone is taken to obey a functional form similar to the elasto-viscoplastic formulation used to model the bulk material.

To the authors' knowledge a rigorous discussion on the merit and fallacies of each of the above mentioned general approaches has not been tackled. It is evident that the dissipation occurring in the real material has to be accounted for in any realistic modelling attempt, so the problem eventually condenses to whether it is possible to neglect the dissipation occurring at the interface, capturing the overall behaviour by simply focusing on the bulk material (as for example attempted with analytical tools by Xu et al. [19] and Persson and Brener [20]). In our opinion the answer is negative. In the cohesive-zone approach the zero-thickness interface is assigned a mechanical behaviour which indeed originates from the interaction of the crack with a process zone which is possibly very thin, yet of finite thickness. This region of finite measure is "lumped" into a line (or a surface in 3D) and hence it seems to be necessary to account for its own time-dependence. It is interesting to compare this observation with the experimental work of Hauch and Marder [21]. They observed how the increase in fracture energy with crack speed was matched by the development of a microstructure of branching transversal cracks whose length and density was increasing too. They also noted though that below a certain speed the fracture energy was still not constant in spite of the lack of any observable transversal crack pattern. Upon dissection of the specimens it was found out that the additional dissipation was likely to be connected with some "subsurface activity", in their terminology. We postulate that these occurences can only be accounted for by introducing the 
rate-dependence at the interface level. Also it is worth noting that this approach has a very significant advantage for the practising engineer. In many structures elastic materials are bonded using polymers, and the analysis could avoid modelling the polymeric layer altogether, under certain restrictions, replacing it with interface elements.

As for the use of plasticity, it might be deemed not physically justified as it can hardly be evoked to explain that tractions decrease to zero at incipient fracture. It is possibly an effective numeric tool to reproduce softening but, as crack formation and/or propagation ultimately involves breaking bonds at the molecular or atomic scale, it seems to be better described by damage mechanics from the physical point of view.

In this paper we aim to capture the rate dependence within the process zone of the interface itself regardless of the behaviour of the surrounding material, and we strive to do so resorting to first principles. It is clear that assumptions are necessary to model such a complex phenomenon as fracture, yet our aim is to develop a general, physically well based cohesive model, without resorting to any phenomenological law other than basic physical and engineering understanding.

As a cornestone of our modelling approach we recognize the existence of an intrinsic, i.e. rate-indipendent, fracture energy. This is related to an elastic energy threshold needed to break bonds at the micro or possibly the atomistic scale [22]. We then use a damage-mechanics approach and introduce a suitably defined damage variable whose evolution is related to the difference between the energy threshold and the elastic energy. The rate-dependence of the overall dissipated energy during crack propagation is a natural by-product of the visco-elastic dissipation lumped on the zero-thickness interface. We postulate that a reasonable characterization of the interface rate-dependence can be achieved by assuming that the interfaces behaves, in a suitably defined way, as its constituent material considered as a continuum. We then present the cohesive model formulation in the general framework of thermodynamics.

To validate the concept we (i) specialise our formulation to the case of a rubber interface, (ii) make the simplest possible assumption by assuming a quadratic form for the elastic energy and by choosing a linear viscoelastic law with exponential kernel and one 
relaxation time only and (iii) present a comparative analysis of numerical and experimental results.

The structure of the paper is as follows. In Section 2, starting from fundamental physical principles we derive a thermodynamically consistent formulation of our proposed interface model and obtain the governing equations. The algorithmic implementation is described in Section 3. Numerical results are then compared with experimental findings in Section 4. Conclusions are finally drawn in Section 5 together with a discussion on some of the opened perspectives.

\section{FORMULATION OF THE INTERFACE MODEL}

We postulate that the interface response can be described by resorting to the free energy potential of the material considered as a continuum. The functional dependence of the free energy upon its arguments is kept the same as in the continuum case while the variables themselves are suitably adapted. This is a result of the dimension reduction implied by the use of a cohesive-zone model, whereby a thin layer is modelled as a surface in 3D and a two dimensional slender region is shrunk into a line in 2D. Accordingly, displacement discontinuity jumps at the interface replace continuum strains as new deformation measures. Progressive degradation of the interface and its ultimate failure is then reproduced naturally through a damage variable. No ad hoc phenomenon is invoked to explain the additional dissipation other than the dissipative mechanisms inherent to the material. More specifically, we assume the damage law to be rate-independent and to be driven by the specific elastic energy.

In general, the traction $\sigma$ at time $t$ at a point of an interface with memory is characterized by a functional $\mathcal{F}$ defined on a suitable set of displacement jump histories $\delta:]-\infty, t] \rightarrow \Re^{n}:$

$$
\boldsymbol{\sigma}(t)=\mathcal{F}(\boldsymbol{\delta})
$$

where $n$ denotes the number of components of $\boldsymbol{\delta}$ (and $\boldsymbol{\sigma}$ ). In the framework of thermodynamics with internal variables, we consider all the past history as "condensed" in 
suitably defined internal variables, which together with the so-called observable variables [10] fully define the state of the material.

The free energy $\Psi$ can then be expressed as

$$
\Psi=\Psi(\boldsymbol{\delta}, \boldsymbol{\alpha}, \mathbf{D})
$$

where $\delta$ represents the displacement discontinuity jump, $\boldsymbol{\alpha}$ denotes internal variables associated with the rate-dependence of the mechanical response and $\mathbf{D}$ represents an internal variable too, which is grouped separately as it exclusively describes the damaging process. In a general case, $\delta, \alpha$ and $\mathrm{D}$ are vectors and therefore they have been denoted with boldface characters.

Following the classic definition of damage due to Kachanov [23] it is suggested that $\Psi$ will depend linearly on $\mathbf{D}$, which is assumed to be $m$-dimensional:

$$
\Psi(\boldsymbol{\delta}, \boldsymbol{\alpha}, \mathbf{D})=(\mathbf{1}-\mathbf{D}) \cdot \tilde{\Psi}(\boldsymbol{\delta}, \boldsymbol{\alpha})
$$

where $\tilde{\boldsymbol{\Psi}}$ denotes a suitably defined vector of free-energy components, i.e. $\tilde{\boldsymbol{\Psi}}^{T}=\left[\begin{array}{llll}\Psi_{1} & \Psi_{2} & \ldots & \Psi_{m}\end{array}\right]$, ' $'$ ' indicates the scalar product and $\mathbf{1}$ is the $m$-dimensional vector $\mathbf{1}^{T}=\left[\begin{array}{llll}1 & 1 & \ldots & 1\end{array}\right]$.

The choice of the number and type of the internal variables depends of course on the phenomenon being addressed. The great flexibility and power of the procedure lies in that these variables can be chosen to represent a vast range of physical phenomena occurring within the material.

In this paper attention is devoted to mode-I crack propagation and therefore the displacement jump specialises to a scalar $\delta$. Also, we deem it appropriate, for the purposes of the proof of concept, to initially present results obtained resorting to a very simple timedependent law, with only one characteristic time. Accordingly, the rheological model represented in Figure 1 is introduced and a single scalar $\alpha$ is used as internal variable, which denotes the inelastic displacement jump in the viscous unit.

The following relation between total (observable) displacement jump $\delta$ and the internal variable $\alpha$ is stated 


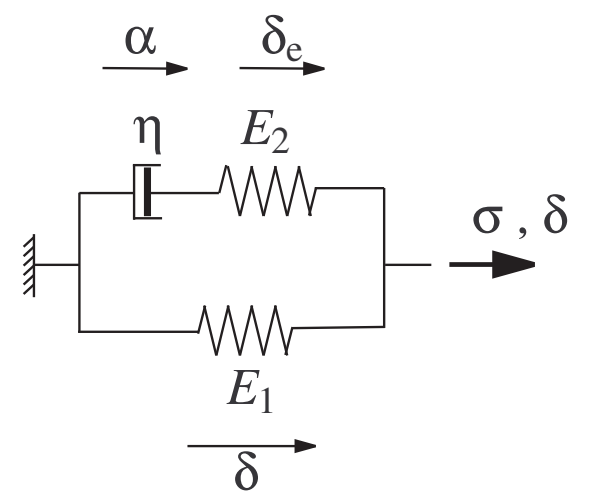

Figure 1: Reological representation of the model

$$
\delta=\delta_{e}+\alpha
$$

where $\delta_{e}$ represents the internal elastic displacement jump within the inelastic arm.

We choose the following expression for the free energy introducing a (formally) twocomponent damage vector $\mathbf{D}^{T}=\left[\begin{array}{ll}D_{1} & D_{2}\end{array}\right]$ (i.e. $m=2$ ):

$$
\Psi=\Psi(\delta, \alpha, \mathbf{D})=(\mathbf{1}-\mathbf{D}) \cdot\left[\begin{array}{c}
\frac{1}{2} E_{1}<\delta>_{+}^{2} \\
\frac{1}{2} E_{2}<\delta-\alpha>_{+}^{2}
\end{array}\right]+K_{c} \mathbf{1} \cdot\left[\begin{array}{c}
\frac{1}{2} E_{1}<\delta>_{-}^{2} \\
\frac{1}{2} E_{2}<\delta>_{-}^{2}
\end{array}\right]
$$

where symbols $<\bullet>_{+}$and $<\bullet>_{-}$represent the positive and negative parts of the argument, respectively, while $E_{1}$ and $E_{2}$ are the elastic stiffness values in the elastic and the inelastic arm of the rheological model. The second term at the RHS in the above equation reflects the further assumptions that, in compression, damage does not influence the response due to crack closure, rate-dependence is negligible and the interface stiffness is amplified by a scalar $K_{c}$. Notice that the last two assumptions are physically justified by the typical behaviour of rubber under confined compression [24].

\subsection{Thermodynamical Consistency}

The definition of evolution laws for the internal variables cannot be established without ensuring such laws do not violate the second principle of thermodynamics for any 
arbitrary process, following the rational mechanics approach introduced by Coleman and Noll [25].

We assume the process is isothermal and, by considering the explicit free-energies of interest in the Clausius-Duhem inequality, in Section 7.1 of the Appendix it is shown that the following conditions are sufficient for thermodynamical consistency:

$$
\left\{\begin{array}{l}
\sigma=(\mathbf{1}-\mathbf{D}) \cdot\left[\begin{array}{c}
E_{1}<\delta>_{+} \\
E_{2}<\delta-\alpha>_{+}
\end{array}\right]+K_{c} \mathbf{1} \cdot\left[\begin{array}{c}
E_{1}<\delta>_{-} \\
E_{2}<\delta>_{-}
\end{array}\right] \\
\frac{\partial \tilde{\Psi}_{2}}{\partial \alpha} \dot{\alpha}=E_{2} \dot{\alpha}<\delta-\alpha>_{+} \geq 0 \\
\dot{D}_{1}, \dot{D}_{2} \geq 0 \\
D_{1}, D_{2} \leq 1
\end{array}\right.
$$

where $\sigma$ denotes the normal interface stress.

\subsection{Internal Variables Evolution Law}

Having established the thermodynamical constraints for the internal variables, complying evolution laws can be chosen. With regards to the internal variable $\alpha$, a simple candidate equation capable of fulfilling Equations (6) is then found in:

$$
\dot{\alpha}=\frac{\sigma-\sigma_{1}}{\eta}
$$

where $\sigma_{1}$ denotes the stress in the elastic arm of the rheological model and $\eta$ is a positive constant. With this choice linear visco-elasticity with an exponential kernel is recovered. This law is considered sufficiently accurate to model the process zone in order to capture the essential aspects of the time-dependent response within an expected range of validity. It has to be stressed however that the presented formulation can be easily enriched using more complex viscous laws.

As far as $\mathbf{D}$ is concerned, it has be noted that a characterization of its evolution should rely on the availability of a micromechanical argument. In more detail, once attention is directed towards a specific material, micromechanics might suggest different evolution 
laws to be tailored for the components of $\mathbf{D}$. For the purposes of the algorithm presentation it is assumed without loss of generality that $D_{1}=D_{2}$, reducing hence $\mathbf{D}$ to a scalar variable $D$.

For the evolution of $D=D_{1}=D_{2}$ the law proposed in Ref. [26] is chosen, as a result of the fact it reflects our assumption that damage is driven solely by local elastic energy.

An energy threshold for damage growth $Y_{c}=Y_{c}(D)$ is introduced and the following Khun-Tucker constraints are then obtained:

$$
Y \leq Y_{c} \quad \dot{D} \geq 0 \quad\left(Y-Y_{c}\right) \dot{D}=0
$$

where

$$
Y=\frac{\partial \Psi_{1}}{\partial \delta}
$$

It is important to underline the choice made on the damage-driving function $Y$, which does not depend on $\Psi$ but only on $\tilde{\Psi}_{1}$. This decision is motivated by rubber's microstructure. In more detail, the attempt of using linear visco-elasticity to model rubber could rely on assuming that its free energy is the sum of an elastic contribution and viscous contribution. The former is thought to be related to the stretch and distorsion of the load-bearing network, while the latter originates from entanglements and motions of parts of the network that are deforming with the main load-bearing network as a result of topological constraints (sulphur bonds as a first example), more details can be found in [27]. Within this conceptual scheme we notice that, as fracture is caused by failure in the load-bearing network, the damage variable has to be driven by its related energy exclusively.

Hence, the following relationship between $Y_{c}$ and $D$ is assumed

$$
Y_{c}=\left\{\begin{array}{lll}
G_{0} & \text { if } & D=0 \\
\frac{G_{0}}{\left(1-\frac{G_{c}-G_{0}}{G_{c}} D\right)^{2}} & \text { if } & 0 \leq D \leq 1 \\
\bar{Y}_{c} & \text { if } & D=1
\end{array}\right.
$$

where $G_{c}$ is the intrinsic fracture energy, $G_{0}$ the elastic energy threshold below which there cannot be damage growth and $\bar{Y}_{c}=\max _{\tau \leq t} Y$ is the maximum of $Y$ over the previous history. Notice that Equation $(10)_{3}$ is introduced for completeness, so that Equation 
$(8)_{1}$ continues to be satisfied, but it only has a formal importance, because when $D=1$ cohesion is lost and no more energy is dissipated. It was shown in Ref. [26] that this relationship, when used in conjunction with a free energy of the form $\Psi(\delta, D)=\frac{1}{2}(1-D) E \delta^{2}$, results in the widely used bilinear traction-discontinuity jump law. Also, it was shown in [26] how the proposed relationship between $Y_{c}$ and $D$ is equivalent to stating that

where $\delta_{\max }$ is defined as follows:

$$
\begin{array}{ll}
D= \begin{cases}0 & \text { if } \delta_{\max } \leq \delta_{0} \\
\min \left\{1,\left(\frac{G_{c}}{G_{c}-G_{0}}\right)\left(1-\frac{\delta_{0}}{\delta_{\max }}\right)\right\} & \text { if } \delta_{\max } \geq \delta_{0}\end{cases} \\
\text { is defined as follows: }
\end{array}
$$

$$
\delta_{\max }=\delta_{\max }(t)=\max _{\tau \leq t} \delta(\tau)
$$

and $\delta_{0}=\sqrt{2 G_{0} / E_{1}}$ is the value of the displacement jump at which damage starts growing in the elastic arm.

Notice that $Y$ is path independent because it only depends on the current value of the relative displacement, whereby the energy eventually dissipated in the elastic arm of the rheological model, after complete decohesion, is always equal to $G_{c}$. Instead, the total energy dissipated does depend on the whole displacement history $\delta:]-\infty, t] \rightarrow \Re$, because it is the sum of $G_{c}$ and of the the energies dissipated in the spring and in the damper of the inelastic arm. This introduces rate-dependence in the simulated dissipative process within the process zone at the interface.

The proposed model reproduces a bilinear traction-separation law only in the extreme cases of instantaneous and infinitely slow loading, in total analogy with how linear viscoelasticity reduces to linear elasticity in the fast and slow rate limit.

The time-integration algorithm required to solve the obtained governing equations is described in the next section.

\section{ALGORITHMIC IMPLEMENTATION}

This section describes how the constitutive equations of the interface are solved during an incremental step, in the context of a general displacement-based finite element method. 
Limiting the discussion for the sake of simplicity to a specific integration point, the problem essentially consists of determining the traction $\sigma(t)$ corresponding to a given displacement-jump history $\delta:]-\infty, t] \rightarrow \Re$. Without loss of generality we assume that $\lim _{t \rightarrow-\infty} \delta(t)=0$, whereby the problem consists in solving the following system of differential equations in the unknown functions $\sigma(t), D\left(\delta_{\max }\right)$ :

$$
\left\{\begin{array}{lr}
\sigma(t)=\left[1-D\left(\delta_{\max }(t)\right)\right]<\int_{-\infty}^{t} \mathcal{J}(t-\tau) \dot{\delta}(\tau) d \tau>_{+}+\left[1-D\left(\delta_{\max }(t)\right)\right] E_{1}<\delta>_{+} \\
+K_{c}\left(E_{1}+E_{2}\right)<\delta>_{-} \\
D\left(\delta_{\max }\right)=\left\{\begin{array}{lr}
0 & \text { if } \delta_{\max } \leq \delta_{0} \\
\min \left\{1,\left(\frac{G_{c}}{G_{c}-G_{0}}\right)\left(1-\frac{\delta_{0}}{\delta_{\max }}\right)\right\} & \text { if } \delta_{\max } \geq \delta_{0}
\end{array}\right. \\
\delta_{\max }=\max _{\tau \leq t} \delta(\tau)
\end{array}\right.
$$

where $\mathcal{J}$ is the relaxation function, defined by

$$
\mathcal{J}(s)=E_{2} e^{-\frac{s}{\lambda}}
$$

and $\lambda$ is the relaxation time $\lambda=\eta / E_{2}$.

Equations (13) cannot be solved by analytical means in the general case and a numerical integration scheme has to be employed. Evidently all the difficulty arises from Equation $(13)_{1}$, as Equation $(13)_{2}$ is an algebraic equation and Equation $(13)_{3}$ can be easily implemented.

Equation (13) $)_{1}$ is approximated by adapting a widely used recursive algorithm [28]. Assuming (again without loss of generality) that the displacement-jump history is nihil before $t=0$ the time interval over which the computation is to be carried out, $[0, T]$, is discretized into a partition of $N$ time increments:

$$
[0, T]=\bigcup_{n=1}^{N}\left[t_{n}, t_{n+1}\right] \quad t_{n+1}=t_{n}+\Delta t_{n}
$$

In the $n$-th time step, the times at the start and the end of the increment are $t_{n}$ and $t_{n+1}$, respectively. 
Therefore, using also Equation (14), the integral in equation (13) $)_{1}$ can be evaluated for $t=t_{n+1}$ as follows:

$$
\int_{-\infty}^{t_{n+1}} \mathcal{J}\left(t_{n+1}-\tau\right) \dot{\delta}(\tau) d \tau=E_{2}\left(\int_{-\infty}^{t_{n}} e^{-\frac{t_{n+1}-\tau}{\lambda}} \dot{\delta}(\tau) d \tau+\int_{t_{n}}^{t_{n+1}} e^{-\frac{t_{n+1}-\tau}{\lambda}} \dot{\delta}(\tau) d \tau\right)
$$

By approximating $\dot{\delta}$ with its finite-difference expression

$$
\dot{\delta}=\frac{\delta\left(t_{n+1}\right)-\delta\left(t_{n}\right)}{\Delta t_{n}}
$$

and using the properties of the exponential functions, one obtains:

$$
\begin{aligned}
& \frac{1}{E_{2}} \int_{-\infty}^{t_{n+1}} \mathcal{J}\left(t_{n+1}-\tau\right) \dot{\delta}(\tau) d \tau= \\
& =e^{-\frac{\Delta t_{n}}{\lambda}} \int_{-\infty}^{t_{n}} e^{-\frac{t_{n}-\tau}{\lambda}} \dot{\delta}(\tau) d \tau+\frac{\delta\left(t_{n+1}\right)-\delta\left(t_{n}\right)}{\Delta t_{n}} \int_{t_{n}}^{t_{n+1}} e^{-\frac{t_{n+1}-\tau}{\lambda}} \dot{\delta}(\tau) d \tau \\
& =e^{-\frac{\Delta t_{n}}{\lambda}} \delta_{e}\left(t_{n}\right)+\left[\delta\left(t_{n+1}\right)-\delta\left(t_{n}\right)\right] \frac{1-e^{-\frac{\Delta t_{n}}{\lambda}}}{\frac{\Delta t_{n}}{\lambda}}
\end{aligned}
$$

The solution scheme proceeds iteratively, so it sufficies to describe one iteration step. At the beginning of a generic time step $n$ the following quantities are known: $\delta_{n}=\delta\left(t_{n}\right)$, $\delta_{e, n}=\delta_{e}\left(t_{n}\right), \delta_{\max , n}=\delta_{\max }\left(t_{n}\right)$ and $D_{n}=D\left(t_{n}\right)$. Their knowledge completely defines the state of the interface. Given a tentative relative displacement $\delta_{n+1}=\delta\left(t_{n+1}\right)$ all the pertinent quantities are then updated as follows

$$
\left\{\begin{array}{l}
\delta_{\max , n+1}=\max \left(\delta_{\max , n}, \delta_{n+1}\right) \\
D_{n+1}=\max \left\{D_{n}, \min \left\{1,\left(\frac{G_{c}}{G_{c}-G_{0}}\right)\left(1-\frac{\delta_{0}}{\delta_{\max , n+1}}\right)\right\}\right\} \\
\sigma_{n+1}=\left(1-D_{n+1}\right) E_{2}<\left[e^{-\frac{\Delta t_{n}}{\lambda}} \delta_{e, n}+\frac{1-\frac{-\Delta t_{n}}{\frac{\Delta t_{n}}{\lambda}}}{\lambda}\left(\delta_{n+1}-\delta_{n}\right)\right]>_{+}+ \\
+\left(1-D_{n+1}\right) E_{1}<\delta_{n+1}>_{+}+K_{c}\left(E_{1}+E_{2}\right)<\delta_{n+1}>_{-}
\end{array}\right.
$$

The algorithm and its associated tangent operator, reported in Section 7.2 of the Appendix, have been implemented as the constitutive law of interface elements within an in-house non-linear finite-element code, which has been used to conduct the numerical simulation discussed in the next section. 


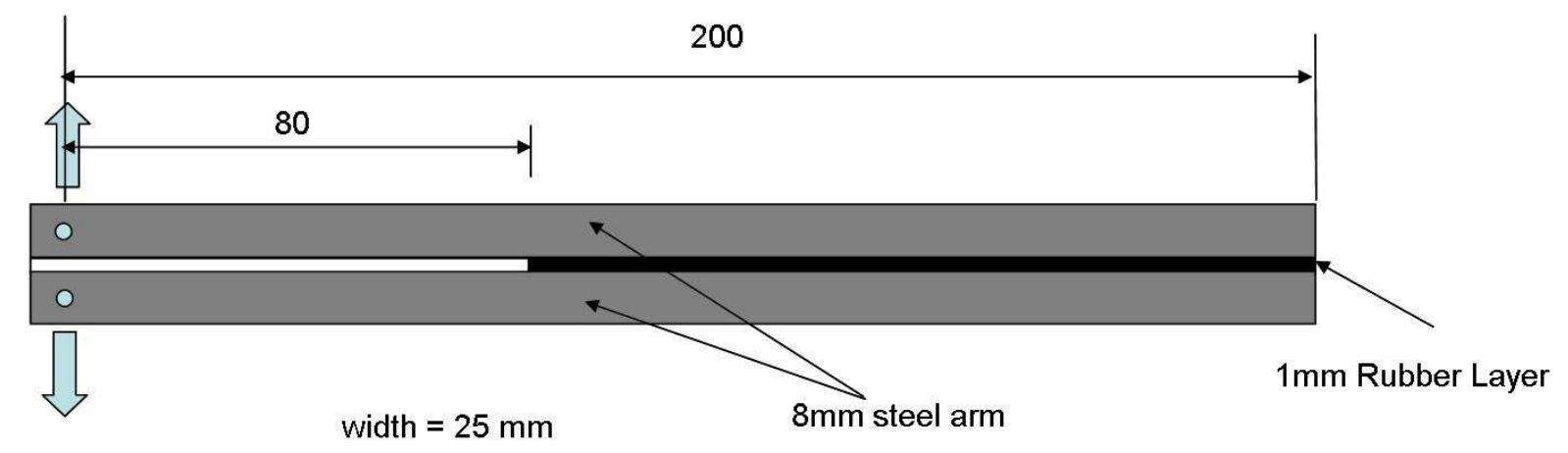

Figure 2: Geometry and loading of the tested DCB specimen.

\section{ANALYSIS OF EXPERIMENTAL AND NUMERICAL RESULTS}

A 25mm wide double-cantilever-beam (DCB) specimen made of $8 \mathrm{~mm}$ thick steel arms separated by a $1 \mathrm{~mm}$ thick interface of SBR / NR blend rubber has been tested under displacement control and at six different rates of the monotonically increasing prescribed cross-head displacement, equal to $0.01,0.1,1,10,100$ and $500 \mathrm{~mm} \mathrm{~min}^{-1}$. The geometry of the specimen is sketched in Figure 2. A close-up of the delamination front is presented in Figure 3.

For the numerical analysis a Young's modulus of $210 \mathrm{GPa}$ and a Poisson's ratio of 0.3 have been used for the steel arms. For the interface the constants reported in the Table 1 have been used. The first three parameters in the table have been calibrated based on the test speed of $100 \mathrm{~min} \mathrm{~mm}^{-1}$. For the relaxation time and the ratio between $E_{2}$ and $E_{1}$ the additional test speed of $10 \mathrm{~min} \mathrm{~mm}^{-1}$ has been used.

Due to simmetry with respect to the crack plane only the top half of the specimen was modelled. The metallic arm was meshed using a single row of 100 8-node (i.e. quadratic) plane-strain fully-integrated elements, each one being $2 \mathrm{~mm}$ long. The rubber interface was modelled using 60 6-node interface elements, of the same size, with 3 integration points and a Newton-Cotes integration rule. Of course the $2 \mathrm{D}$ approximation cannot 
(a)

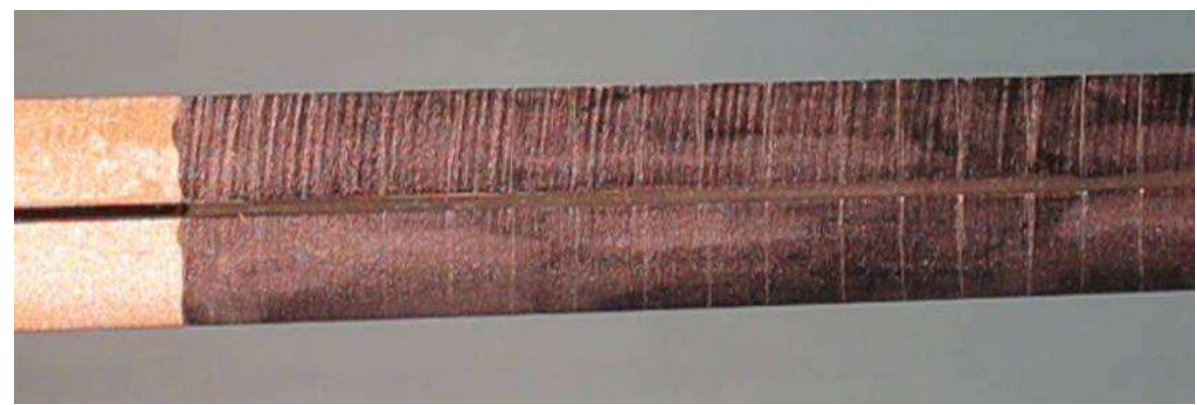

(b)

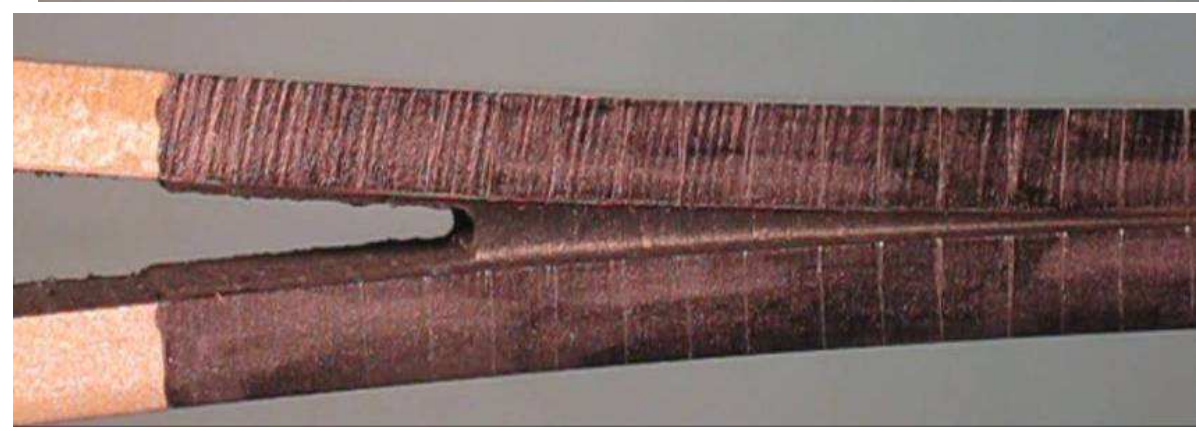

Figure 3: Experimental test: particular of the (a) undeformed and (b) deformed specimen.

\begin{tabular}{ccccc}
\hline$G_{c}\left(\mathrm{~N} \mathrm{~mm}^{-1}\right)$ & $G_{0}\left(\mathrm{~N} \mathrm{~mm}^{-1}\right)$ & $E_{1}\left(\mathrm{~N} \mathrm{~mm}^{-3}\right)$ & $E_{2}\left(\mathrm{~N} \mathrm{~mm}^{-3}\right)$ & $\lambda(\mathrm{s})$ \\
\hline 9.5 & 3.8 & 33 & 27 & 250 \\
\hline
\end{tabular}

Table 1: Input parameters used for the rubber interface

capture the fact that the crack front is not a straight line, but it was shown in Ref [29] that in general this leads to reasonably accurate results for wide enough specimen, such as the one tested in our case.

Figure 4 shows the experimental and the numerical load-displacement curves for the case of test speed of $100 \mathrm{~min} \mathrm{~mm}^{-1}$ and shows that the numerical model can correctly capture all aspects of the experimental behaviour, with good agreement both qualitatively and quantitatively. In this figure five points of the debonding process have been marked with the letters $a, b, c, d$ and $e$, to identify the following five essential parts or moments of the debonding process: (a) elastic loading, (b) onset of fracture, (c) self-similar crack propagation, (d) disruption of self-similarity due to the crack-tip field reaching the specimen boundary and (e) almost-rigid rotation following the almost complete propagation of the crack. 


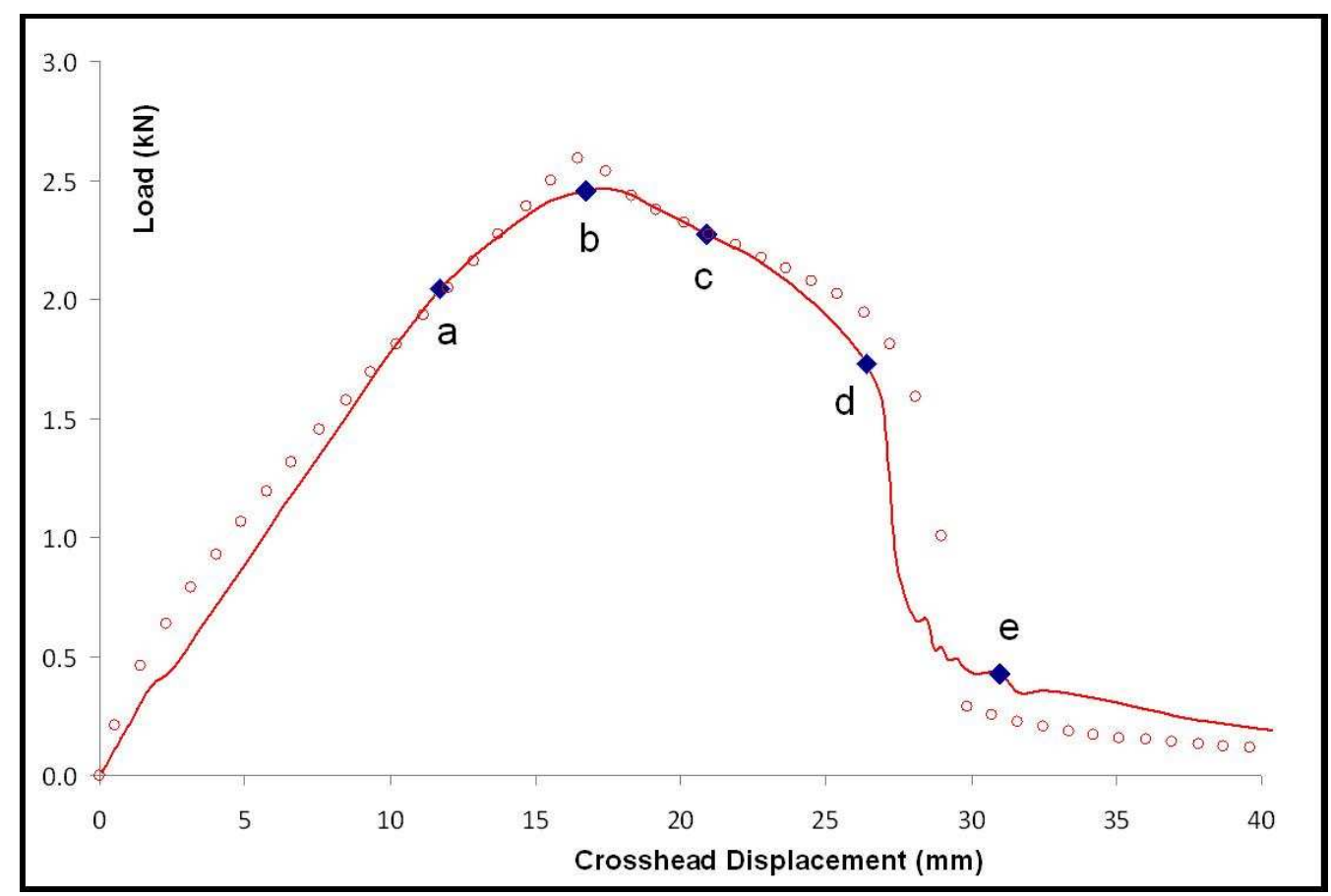

Figure 4: Numerically and experimentally obtained load-displacement curves for the test speed of $100 \mathrm{~min}$ $\mathrm{mm}^{-1}$, showing the points of the debonding process referred to in Figure 5.

The direct stress in the vertical direction across the steel arm $\left(\sigma_{y y}\right.$ in the model reference system) has been chosen as a reasonable indicator of the process-zone activity and Figures 5.(a)-(e) show the contour plots of $\sigma_{y y}$ at the points of the debonding process indicated in Figure 4. At point $a$ (elastic loading), a stress concentration is forming at the crack tip: the interface discontinuity-jump, albeit non zero, is 'small', and the stress profile resembles the singularity field characteristic of classical continuum elements. At point $b$, the crack has just started propagating, causing the stress distribution to be more spread. At point $c$ the crack is now moving in a self-similar way. This is happening on a rather limited length due to the relatively large size of the process zone in relation to the sample. At point $d$ the compression distribution at the far left side is changing, as the lack of available space and the need of maintaining the overall resultant bending moment lead to a narrower and more intense stress distribution. Finally, at point $e$ debonding is almost complete, the arm can only sustain negligible bending and the total force has now reduced to almost zero. 
(a)

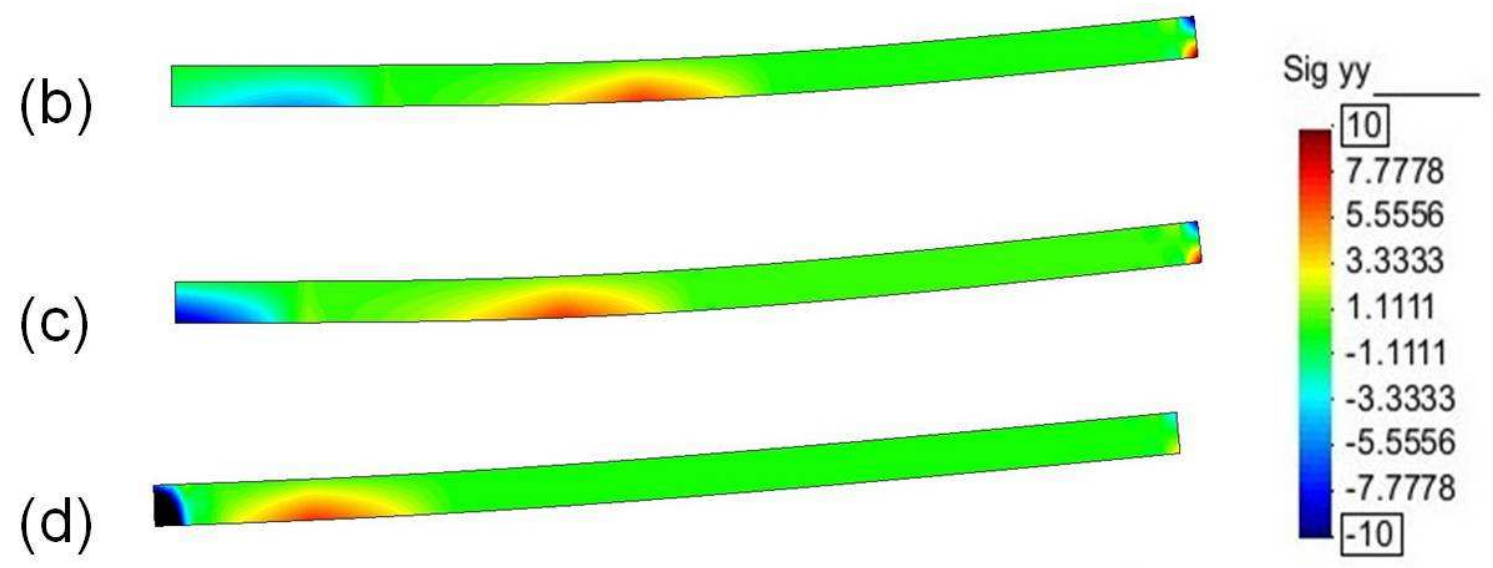

(e)

Figure 5: Contours plots of the direct stress in the vertical direction at the points of the debonding process indicated in Figure 4. 
Figure 6 shows a comparison of the numerical and experimental load-displacement curves for all the considered test speeds. Therefore, their analysis allows validating the capability of the model to capture rate dependence. The numerical curve obtained at 500 $\min \mathrm{mm}^{-1}$ has not been reported because it practically overlaps with the one obtained at $100 \mathrm{~min} \mathrm{~mm}^{-1}$. The model prediction is satisfactory only on a range of applied displacement rates. More in detail, it can be seen that the model does not capture the change of the curve when the applied rate is increased from $100 \mathrm{~min} \mathrm{~mm}^{-1}$ to $500 \mathrm{~min} \mathrm{~mm}^{-1}$. The same happens at the low range of speed, indeed the computed response at $0.1 \mathrm{~min}$ $\mathrm{mm}^{-1}$ is rather close to the computed response at $0.01 \mathrm{~mm} / \mathrm{min}$, while the experimental response is characterized by lower global loads. This lack of ability to predict the rate dependence over 5 logarithmic decades of applied displacement rate is not surprising at all. The same qualitative behaviour can be seen in similar visco-elastic analyses if the relaxation spectrum employed in the costituive model is too narrow with respect to the real material. This is certainly our case, as we are using a discrete spectrum with a single relaxation time to model rubber behaviour, whose spectrum is not only continuous but rather wide too, spanning no less than 5 logarithmic decades.

On the other hand, a visco-elastic model with one relaxation time is expected to capture relatively well the rate dependence roughly over two logarithmic decades. This is indeed what happens in our model, which is an extremely promising result, because it indicates that the use of a wider relaxation spectrum or of a more complex, possibly nonlinear, viscoelastic law is expected to improve the predicitve capacity without substantially altering the conceptual framework herein presented.

\section{Conclusions}

A novel cohesive-zone model suitable for the simulation of rate-dependent crack propagation along interfaces has been presented. Recognizing the existence of an intrinsic, rate-indipendent, fracture energy and introducing damage as the most physically appropriate internal variable to reproduce fracture, a consistent thermodynamical formulation is obtained. It is postulated that the rate-dependent dissipation on the interface is a re- 


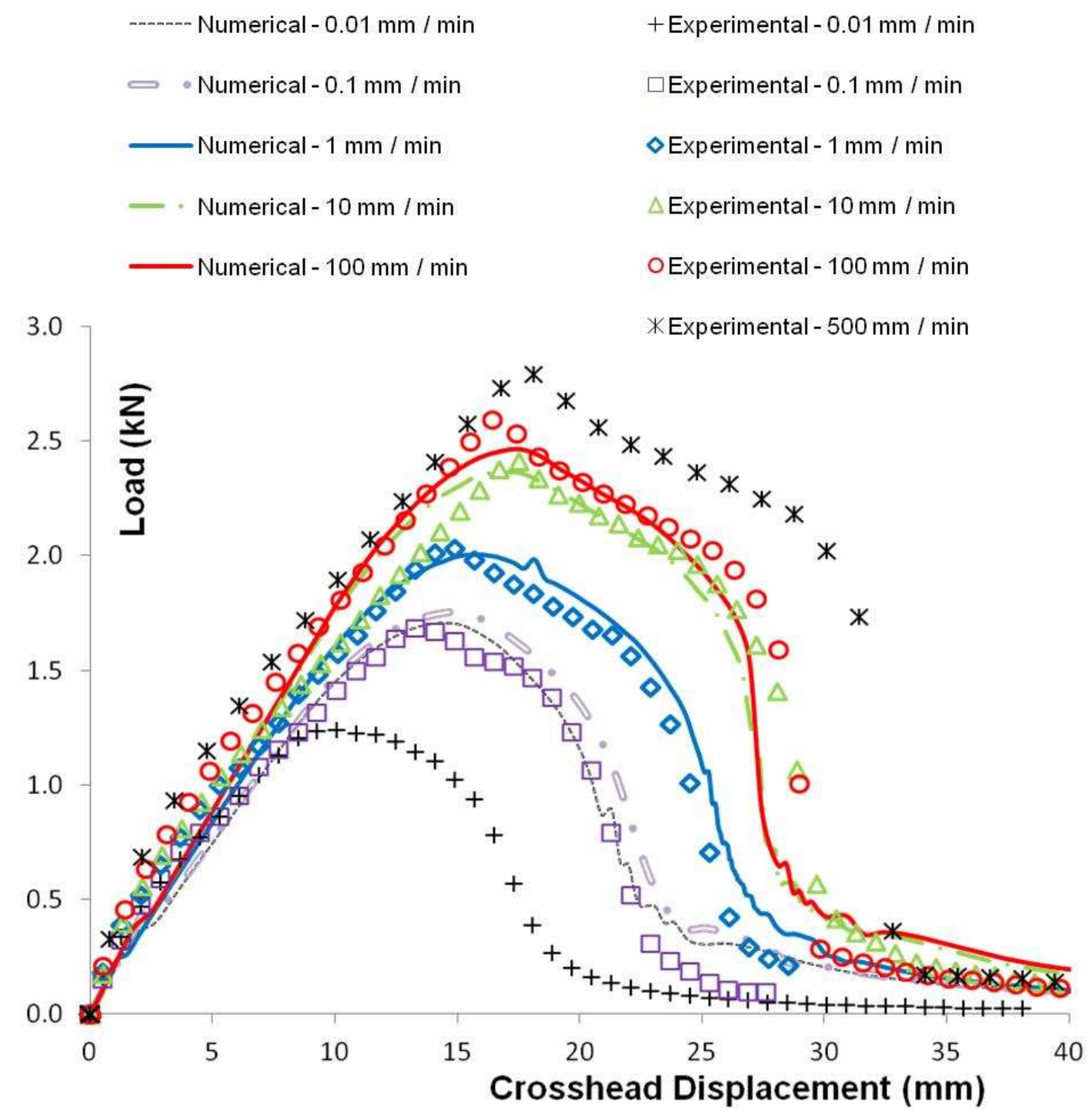

Figure 6: Experimental vs. numerical load - deflection curves for the six considered test speeds. 
sult of the same mechanisms observable in the interface material when considered as a continuum. In this way, the procedure reproduces the rate dependence of the 'measured' fracture energy, i.e. of the total energy dissipated within the real (finite-thickness) interface, naturally without further phenomenological assumptions.

The model was specialized to linear visco-elasticity, considered to provide a sufficiently accurate constitutive law for the simulation of the experimental response within a (predictable) range of loading rates and, at the same time, a simple enough law to focus on the essence of the concept, whose proof of validity was the ultimate aim of this work. In the same spirit the model has been specialised to pure mode-I only.

The comparison of the numerical predictions with the experimental results is very satisfactory in this respect, because in the expected range of validity of the model, i.e. the two logaritmic decades of loading rates typically captured by a linear visco-elastic model with one relaxation time, substancially good agreement was obtained. In fact, in addition to the rate dependence, within this interval all the essential features of the experimental results are reproduced with good agreement.

The loss of accuracy at the extremities of the interval of considered test speeds is discussed, pointing at the most natural solution. Since in our opinion the general framework has been successfully established, we are optimistic that future work can lead to obtain greater accuracy of the numerical prediction within a much wider range of test speeds, and possibly even at any test speed, by adopting a more refined visco-elastic law for the rubber layer. Future outlook also includes the extension to pure mode II and to mixed-mode and an investigation on how both the constitutive assumptions and the input parameters of the interface model can be determined via experimental testing of the bulk material and/or numerical simulation or simplified analytical modelling at the micromechanical level. Regarding the first point, in the authors' opinion the extension to pure mode-II cases is quite straightforward, the only difference being that the decomposition of the relative displacement into positive and negative parts is not needed. The extention to mixed-mode does not seem to require any alteration of the conceptual framework, but its experimental validation and calibration are expected to be more complex and time consuming. 


\section{ACKNOWLEDGEMENTS}

The financial support by EPSRC is gratefully acknowledged. The first author would also like to thank Mrs. A. Harris and Mr. C. Myshrall for their help with the experimental work and for their technical advice.

\section{References}

[1] G.I. Barenblatt. The mathematical theory of equilibrium cracks in brittle fracture. Advances in Applied Mechanics, 7:55-129, 1962.

[2] A. Hilleborg, M. Modéer, and P.E. Petersson. Analysis of crack formation and crack growth in concrete by means of fracture mechanics and finite elements. Cement and Concrete Research, 6.

[3] A. Giacomini. Size effects on quasi-static growth of cracks. SIAM Journal of Mathematical Analysis, 36(6):1887-1928, 2005.

[4] R. Frassine, M. Rink, and A. Pavan. Viscoelastic effects on the interlaminar fracture toughness of epoxy/carbon fibre. International Journal of Composite Materials, 27:921-933, 1993.

[5] R. Frassine and A. Pavan. Viscoelastic effects on the interlaminar fracture behaviour of thermoplastic matrix composites: I. Rate and temperature dependence in unidirectional pei / carbon-fibre laminates. Journal of Composite Science Technology, 54:193-200, 1995.

[6] R. Frassine, M. Rink, and A. Pavan. Viscoelastic effects on the interlaminar fracture behaviour of thermoplastic matrix composites: II. Rate and temperature dependence in unidirectional peek / carbon-fibre laminates. Journal of Composite Science Technology, 56:1253-1260, 1996.

[7] J. Du, M.D. Thouless, and A.F. YEE. Effects of rate on crack growth in a rubber modified epoxy. Acta Materialia, 48:3581-3592, 2000. 
[8] Siegmund Xu, C. and K. T., Ramani. Rate-dependent crack growth in adhesives II. experiments and analysis. International Journal of Adhesion and Adhesives, 23:1522, 2003.

[9] T.W. Webb and E.C. Aifantis. Crack growth resistance curves and stick-slip fracture. Mechanical Research Communications, 246.

[10] G.A. Maugin. The thermomechanics of nonlinear irreversible behaviors. World Scientific, Singapore, 1999.

[11] D. Maugis. Subcritical crack growth, surface energy, fracture toughness, stick-slip and embrittlement. Journal of Materials Science, 20:3041-3073, 1985.

[12] M.J. Van Der Bosch, P.J.G Schreurs, and M.G.D. Geers. On the development of a 3D cohesive zone element in the presence of large deformations. Computational Mechanics, 42:171-180, 2006.

[13] T. D. Nguyen and S. Govindjee. Numerical study of geometric constraint and cohesive parameters in steady-state viscoelastic crack growth. International Journal of Fracture, 141(1-2):255-268, 2006.

[14] Siegmund Xu, C. and K. T., Ramani. Rate-dependent crack growth in adhesives I. modelling approach. International Journal of Adhesion and Adhesives, 23:9-13, 2003.

[15] A. Corigliano and M. Ricci. Rate-dependent interface models: formulation and numerical applications. International Journal of Solids and Structures, 38(4):547$576,2001$.

[16] D.H. Allen and C.R. Searcy. A micromechanical model for a viscoelastic cohesive zone. International Journal of Fracture, 107:159-176, 2001.

[17] K.M. Liechti and J.D. Wu. Mixed-mode,time-dependent rubber/metal debonding. Journal of the Mechanics and Physics of Solids, 49:1039-1072, 2001. 
[18] C.M. Landis, Pardoen, and J.W T., Hutchinson. Crack velocity dependent toughness in rate dependent materials. Mechanics of Materials, 32:663-678, 2000.

[19] D.B. Xu, C.Y. Hui, and E.J. Kramer. Interface fracture and viscoelastic deformation in finite size specimens. Journal of Applied Physics, 72(8):3305-3316, 1992.

[20] B.N.J. Persson and E.A. Brener. Crack propagation in viscoelastic solids. Physical Review E, 71(3), 2006.

[21] J.A. Hauch and M.P. Marder. Energy balance in dynamic fracture, investigated by a potential drop tecnique. International Journal of Fracture, 90:133-151, 1998.

[22] G.J Lake and A.G. Thomas. The strength of highly elastic materials. Proceedings of the Royal Society, 300:108-119, 1967.

[23] L.M Kachanov. On the Time to Failure under Creep Conditions. Izv.Akad.Nauk.SSR, $8: 26-31,1958$.

[24] M. Destrade, M.D. Gilchrist, J.A. Motherway, and J.G. Murphy. Bimodular rubber buckles early in bending. 42:469-476, 2010.

[25] C. Truesdell and W. Noll. The non-linear field theories of mechanics. vol. iii/3. In S. Flugge, editor, Handbuch der Physik. Vol III/3, Berlin, 1965. Springer.

[26] G. Alfano and M.A. Crisfield. Finite element interface models for the delamination analysis of laminated composites: mechanical and computational issues. International Journal for Numerical Methods in Engineering, 50(7):1701-1736, 2001.

[27] J.S. Bergstrom and M.C Boyce. Large strain time-dependent behavior of filled elastomers. Mechanics of Materials, 32:627, 644, 2000.

[28] K.S.and Goudeau G.L. Taylor, R.L.and Pister. Thermomechanical analysis of viscoelastic solids. International Journal for Numerical Methods in Engineering, 2:4559, 1970. 
[29] Y. Mi, M.A. Crisfield, G.A.O. Davies, and H.B. Hellweg. Progressive delamination using interface elements. Journal of Composite Materials, 32(14):1246-1272, 1998.

[30] B.D. Coleman and M.E. Gurtin. Thermodynamics with internal variables. Journal of Chemical Physics, 47:597-613, 1967.

\section{APPENDIX}

\subsection{THERMODYNAMICAL CONSISTENCY}

We assume that the process is isothermal and therefore the second law specialises [30] to the following:

$$
\sigma \dot{\delta}-\dot{\Psi} \geq 0
$$

For the free energy of our choice

$$
\Psi=\Psi(\delta, \alpha, \mathbf{D})=(\mathbf{1}-\mathbf{D}) \cdot\left[\begin{array}{c}
\frac{1}{2} E_{1}<\delta>_{+}^{2} \\
\frac{1}{2} E_{2}<\delta-\alpha>_{+}^{2}
\end{array}\right]+K_{c} \mathbf{1} \cdot\left[\begin{array}{c}
\frac{1}{2} E_{1}<\delta>_{-}^{2} \\
\frac{1}{2} E_{2}<\delta>_{-}^{2}
\end{array}\right]
$$

an application of the chain rule yields:

$$
\begin{array}{r}
\dot{\Psi}=\left[(\mathbf{1}-\mathbf{D}) \cdot\left[\begin{array}{c}
E_{1}<\delta>_{+} \\
E_{2}<\delta-\alpha>_{+}
\end{array}\right]+K_{c} \mathbf{1} \cdot\left[\begin{array}{c}
E_{1}<\delta>_{-} \\
E_{2}<\delta>_{-}
\end{array}\right]\right] \dot{\delta} \\
+\left[(\mathbf{1}-\mathbf{D}) \cdot\left[\begin{array}{c}
0 \\
-E_{2}<\delta-\alpha>_{+}
\end{array}\right]+K_{c} \mathbf{1} \cdot\left[\begin{array}{l}
0 \\
0
\end{array}\right] \dot{\alpha}\right. \\
-\left[\begin{array}{c}
\frac{1}{2} E_{1}<\delta>_{+}^{2} \\
\frac{1}{2} E_{2}<\delta-\alpha>_{+}^{2}
\end{array}\right] \cdot \dot{\mathbf{D}}
\end{array}
$$

Upon inserting in Equation (20) and factorising $\dot{\delta}$ 


$$
\begin{gathered}
\left\{\sigma-\left[(\mathbf{1}-\mathbf{D}) \cdot\left[\begin{array}{c}
E_{1}<\delta>_{+} \\
E_{2}<\delta-\alpha>_{+}
\end{array}\right]+K_{c} \mathbf{1} \cdot\left[\begin{array}{c}
E_{1}<\delta>_{-} \\
E_{2}<\delta>_{-}
\end{array}\right]\right]\right\} \dot{\delta}+ \\
+\left\{(\mathbf{1}-\mathbf{D}) \cdot\left[\begin{array}{c}
0 \\
E_{2}<\delta-\alpha>_{+}
\end{array}\right]+K_{c} \mathbf{1} \cdot\left[\begin{array}{c}
0 \\
0
\end{array}\right]\right\} \dot{\alpha}+ \\
+\left[\begin{array}{c}
\frac{1}{2} E_{1}<\delta>_{+}^{2} \\
\frac{1}{2} E_{2}<\delta-\alpha>_{+}^{2}
\end{array}\right] \cdot \dot{\mathbf{D} \geq 0}
\end{gathered}
$$

Sufficient conditions for the above inequality to hold are as follows:

$$
\left\{\begin{array}{l}
\sigma=(\mathbf{1}-\mathbf{D}) \cdot\left[\begin{array}{c}
E_{1}<\delta>_{+} \\
E_{2}<\delta-\alpha>_{+}
\end{array}\right]+K_{c} \mathbf{1} \cdot\left[\begin{array}{c}
E_{1}<\delta>_{-} \\
E_{2}<\delta>_{-}
\end{array}\right] \\
\frac{\partial \tilde{\Psi}_{2}}{\partial \alpha} \dot{\alpha}=E_{2} \dot{\alpha}<\delta-\alpha>_{+} \geq 0 \\
\dot{D}_{1}, \dot{D}_{2} \geq 0 \\
D_{1}, D_{2} \leq 1
\end{array}\right.
$$

\subsection{TANGENT OPERATOR}

The consistent tangent stiffness is computed through routine algebraic manipulations as follows:

$$
\frac{\partial \sigma}{\partial \delta}=\left\{\begin{array}{lll}
0 & \text { if } & D=1 \forall \delta \\
(1-D)\left[E_{1}+E_{2} \frac{\lambda}{\Delta t}\left(1-e^{\frac{-\Delta t}{\lambda}}\right)\right]-\frac{\partial D}{\partial \delta} \sigma & \text { if } & D \neq 0 \text { and } \delta \geq 0 \\
K_{c} & \text { if } & D \neq 0 \text { and } \delta \leq 0
\end{array}\right.
$$

